\title{
Reply to the Letter to the Editor: The impact of preoperative hand grip strength on the long-term outcomes after gastrectomy for advanced gastric cancer
}

\author{
Ryota Matsui $^{1,2}$ (1) Noriyuki Inaki ${ }^{1,3} \cdot$ Toshikatsu Tsuji $^{1}$
}

Received: 12 April 2021 / Accepted: 16 April 2021 / Published online: 12 May 2021

(c) Springer Nature Singapore Pte Ltd. 2021

\section{Dear Editor,}

We have read with interest the Letter to the Editor for our original article. We would like to respond to the matters pointed out.

First, as mentioned, the current diagnostic criteria for sarcopenia require a low hand grip strength (HGS) [1]. In the present our study, it is important to note that we considered the HGS and skeletal muscle mass as separate prognostic indicators. If we had described a low skeletal muscle mass as indicative of a low skeletal muscle mass index (SMI) instead of sarcopenia, there might have been less misunderstanding. In many retrospective studies of gastrointestinal cancers, sarcopenia is diagnosed by a low skeletal muscle mass alone. The systematic reviews of postoperative outcomes also defined a low SMI as sarcopenia $[2,3]$. Therefore, it may have been appropriate for the reviewers to refer to a low skeletal muscle mass as sarcopenia.

Second, we reanalyzed the multivariate analysis, including the gender and absolute values of height and weight in addition to the age and showed that a low HGS was an independent poor prognostic factor (hazard ratio $2.735,95 \%$ confidence interval 1.063-7.037, $P=0.037$ ). Our thoughts on the cutoff values for HGS are described in the "Discussion" section. We believe that the cutoff value for postoperative complications is different from that for the long-term prognosis, as postoperative complications occur soon after the preoperative HGS is measured,

Noriyuki Inaki

n.inaki@ viola.ocn.ne.jp

1 Department of Gastroenterological Surgery, Ishikawa Prefectural Central Hospital, 2-1 Kuratsuki-higashi, Kanazawa, Ishikawa 920-8201, Japan

2 Department of Surgery, Juntendo University Urayasu Hospital, 2-1-1 Tomioka, Urayasu 270-0021, Japan

3 Department of Gastroenterological Surgery, Kanazawa University, 13-1 Takara-machi, Kanazawa, Ishikawa 920-8641, Japan but postoperative body weight loss tends to be associated with the long-lasting physiological disorder associated with gastrectomy. Patients without sarcopenia preoperatively reportedly have a poor prognosis if they develop sarcopenia postoperatively [4]. It is natural for patients with a low HGS before surgery to have a poor prognosis, as they have sarcopenia. What is noteworthy in this study is that the prognosis is affected by the setting of a cutoff value that includes patients who are considered healthy.

Third, whether or not HGS can be used as a "vital sign" is unclear, but I agree that it may be a simple and powerful predictor. In the future, we hope to verify the validity of this study based on the preoperative measurements of both the skeletal muscle mass and HGS.

\section{Declarations}

Conflict of interest Ryota Matsui and other co-authors have no conflicts of interest.

\section{References}

1. Cruz-Jentoft AJ, Bahat G, Bauer J, Boirie Y, Bruyère O, Cederholm T, et al. Sarcopenia: revised European consensus on definition and diagnosis. Age Ageing. 2019;48(1):16-31.

2. Yang Z, Zhou X, Ma B, Xing Y, Jiang X, Wang Z. Predictive value of preoperative sarcopenia in patients with gastric cancer: a meta-analysis and systematic review. J Gastrointest Surg. 2018;22(11):1890-902.

3. Kamarajah SK, Bundred J, Tan BHL. Body composition assessment and sarcopenia in patients with gastric cancer: a systematic review and meta-analysis. Gastric Cancer. 2019;22(1):10-22.

4. Kudou K, Saeki H, Nakashima Y, Sasaki S, Jogo T, Hirose K, et al. Postoperative development of sarcopenia is a strong predictor of a poor prognosis in patients with adenocarcinoma of the esophagogastric junction and upper gastric cancer. Am J Surg. 2019;217(4):757-63.

Publisher's Note Springer Nature remains neutral with regard to jurisdictional claims in published maps and institutional affiliations. 